\title{
Breath Volatolomics for Medical Applications. Are We There Yet?
}

\author{
Pierre Hugues Stefanuto, Delphine Zanella and Jean François Focant* \\ Organic and Biological Analytical Chemistry Group, University of Liège, Belgium \\ *Corresponding author: Jean François Focant, Department of Chemistry, Organic and Biological Analytical Chemistry Group, Mo- \\ lecular Systems Research Unit, Quartier Agora, University of Liege, Belgium.
}

To Cite This Article: Pierre Hugues Stefanuto, Delphine Zanella, Jean François Focant, Breath Volatolomics for Medical Applications. Are We There Yet?. 2020 - 11(3). AJBSR.MS.ID.001621. DOI: 10.34297/AJBSR.2020.11.001621.

Received: 眥 November 11, 2020; Published: 阱 December 15, 2020

\section{Opinion}

Over the last few decades, exhaled breath has been keeping constant attention of the medical community as it has often been reported as one of the most promising low invasiveness matrix for potential diagnosis of various diseases [1,2]. Indeed, as the volatile organic compounds (VOCs) contained in the exhaled air of a human being are linked to metabolic pathways taking place in the body, one can expect the VOC fingerprint to be impacted by pathologies, especially in the case of pulmonary diseases, but not only as VOCs are blood-borne chemicals. It is therefore open season for the hunt of exhaled biomarkers in analytical laboratories, and many mavericks have been out chasing these days, with various levels of success. Looking into the scientific literature, because of the heterogenicity in study design, sampling approaches, measurements, and data processing, one can barely find comparable studies and conclude on any robust (sets of) VOCs for specific disease diagnostic. Although existing in other area of biomedical research [3], virtually no guidelines or standardization exist in this specific field of breath volatolomics [4] and that is the major issue.

In breathomics, properly defining the clinical question is the first important step to consider to adequately design a study. Such task is to be addressed jointly by both medical and analytical teams, whom sometimes have first to adopt a common language. This has a direct impact on the analytical procedures to implement but also on the possible outcomes and conclusions of a study. The justness of this first step is often neglected and is at least partly responsible for the limited usability of resulting data sets to answer the original question afterwards.

As always in analytical chemistry, the sampling step is crucial. The selection of the relevant breath fraction (mixed expiratory breath, late expiratory breath, alveolar breath...), the type of potential collection container (polymer bags, syringes, canisters...), and the pre-concentration techniques are to be properly and exhaustively documented as breath collection is challenging and has significant impact on the composition of the sample [5]. Furthermore, complete or sorbent-based selective transfer of samples to the measuring device (e.g., Selected-Ion Flow-Tube Mass Spectrometer, SIFT-MS; or comprehensive two-dimensional gas chromatography time-of-flight high resolution mass spectrometry, GC $\times$ GC-HRTOFMS) [6,7] introduces various levels of analytical biases and sensitivity issues. Such a situation might perfectly be fine to isolate putative biomarkers or chemical profiles to build diagnostic models but must be clearly acknowledged to ensure proper usage of the data.

Next, post-acquisition data analysis itself requires an extra level of care to ensure monitoring and control over this oftenoverlooked critical step. Implementation of basic important criteria such as test set/validation set population sizes and proper handling of confounding factors have to be carried out. All details of the complete data processing workflows including in-depth validation of the statistical models used must be made available. It starts from data alignment, pre-processing data transformation (i.e., normalization, scaling, log transformation), (un)supervised screening, and data reduction using univariate (e.g., Fisher Ratio, ANOVA) and multivariate (e.g., PLS-DA, supervised and unsupervised random forest) [8]. If pattern-recognition statistical techniques, clusterisation, or classification models are brought into play to allow possible imaging of specific profiles after data reduction, they have to be tested for accuracy and robustness. At least receiver operating characteristic (ROC) processes should 
be implemented to assess the diagnosis ability of any putatively identified biomarkers [9]. One has globally to better cultivate our ability to ensure data integrity over the entire data processing journey to provide valuable analytical insights to the medical community and make breathomics move up over the technology readiness levels (TRLs).

In any cases, when searching for disease-specific VOCs produced by subtle changes in specific biochemical pathways, uniformity is mandatory to ensure reproducibility and comparability. Even when the required technical expertise is met, $\mathrm{QA} / \mathrm{QC}$ aspects are rarely considered. Calling on reference materials, analytical validation, or proficiency testing is not common in breathomics but is needed to establish enough robustness in discriminating patient from healthy controls for the approach to be utilized at the clinical level. Global method performances could more easily be estimated by simply using benchmark datasets that would be made available for the analytical developers [10]. Categorizing the level of confidence one can have over putative biomarkers is also of prime interest and solutions for harmonization such as the Metabolomics Standards Initiative (MSI) confidence level scaling system are now available [11]. This contributes to further facilitate the transposition of biomarkers from one study to another, bringing higher level of usability of breath analysis in the medical field.

It will only be at the price of stronger and more implemented robust analytical strategies that breath volatolomics will have a real meaning in medical applications. The potential is significant for early diagnosis, for monitoring response to treatment, and for precision medicine. At some point, it will be required to shift from the usage of highly sophisticated separation science tools to more routine-adapted devices, ensuring rapid, reliable and affordable screening. Moreover, hybrid approaches that combine VOC profiles collected from several samples taken from a same patient [12] could even increase the diagnostic value. Combining such multiple VOC measurements with existing clinical methods (e.g., Fractional exhaled Nitric Oxide FeNO, immunotesting, CT scan, ...) might be the way to go, but a passable road is still to be put through...

\section{Acknowledgement}

None.

\section{Conflict of Interest}

No conflict of interest.

\section{References}

1. Mazzatenta A, Di Giulio C, Pokorski M (2013) Pathologies currently identified by exhaled biomarkers. Respir Physiol Neurobiol 187(1): 128134.

2. Beauchamp J, Davis C, Pleil J (Ed.) (2020) Breathborne biomarkers and the human volatilome. Elsevier.

3. Bossuyt PM, Reitsma JB, Bruns DE, Gatsonis CA, Glasziou PP, et al. (2015) STARD 2015: an updated list of essential items for reporting diagnostic accuracy studies. BMJ 351: h5527.

4. Henderson B, Ruszkiewicz DM, Wilkinson M, Beauchamp JD, Cristescu SM, et al. (2020) A benchmarking protocol for breath analysis: the peppermint experiment. J Breath Res 14(4): 046008.

5. Lawal O, Ahmed WM, Nijsen TME, Goodacre R, Fowler SJ (2017) Exhaled breath analysis: a review of 'breath-taking' methods for off-line analysis. Metabolomics 13(10): 110.

6. Markar SR, Wiggins T, Antonowicz S, Chin ST, Romano A, et al. (2018) Assessment of a noninvasive exhaled breath test for the diagnosis of oesophagogastric cancer. JAMA Oncol 4(7): 970-976.

7. Stefanuto PH, Zanella D, Vercammen J, Henket M, Schleich F, et al. (2020) Multimodal combination of GC $\times$ GC-HRTOFMS and SIFT-MS for asthma phenotyping using exhaled breath. Sci Rep 10: 16159.

8. Di Giovanni N, Meuwis MA, Louis E, Focant JF (2020) Untargeted serum metabolic profiling by comprehensive two-dimensional gas chromatography-high-resolution time-of-flight mass spectrometry. J Proteome Res 19(3): 1013-1028.

9. Schleich FN, Zanella D, Stefanuto PH, Bessonov K, Smolinska A, et al (2019) Exhaled volatile organic compounds are able to discriminate between neutrophilic and eosinophilic asthma. Am J Respir Crit Care Med 200(4): 444-453.

10. Weggler BA, Dubois LM, Gawlitta N, Gröger TM, Moncur J, et al. (2020) A unique data analysis framework and open-source benchmark data set for the analysis of GC×GC software. J Chromatogr A 461721.

11. Viant MR, Kurland IJ, Jones MR, Dunn WB (2017) How close are we to complete annotation of metabolomes? Curr Opin Chem Biol 36: 64-69.

12. Broza YY, Mochalski P, Ruzsanyi V, Amann A, Haick H (2015) Hybrid volatolomics and disease detection. Angew Chem Int Ed Engl 54(38): 11036-11048 